\title{
大环分子衍生物在人工跨膜离子通道领域中的研究进展
}

\author{
间腾飞 $a, b$ 刘盛达 $a, b$ 罗逸尘 ${ }^{b}$ 邹应萍 $*, a$ 刘俊秋 $*, b, c$ \\ ( ${ }^{a}$ 中南大学化学化工学院 长沙 410083) \\ ( $b$ 杭州师范大学材料与化学化工学院 杭州 310036) \\ ( ${ }^{c}$ 吉林大学化学学院 超分子结构与材料国家重点实验室 长春 130012)
}

\begin{abstract}
摘要 大环分子如环糊精、冠醚等因具备独特的空腔结构, 能够通过主-客体相互作用实现对分子、离子的特异性识别, 因此常被应用于人工跨膜离子传输系统的研究和开发. 与天然的通道蛋白相比, 大环分子具有价格低廉、性质稳定、 结构易于修饰和功能化等诸多优点, 因而备受科研工作者们的关注. 本综述详细介绍了大环分子在人工离子通道领域 中的研究进展，按照不同的大环分子进行分类，系统归纳了基于大环结构的人工跨膜通道的制备方法、结构调控以及 其在生物医药领域中的潜在应用. 最后对大环类人工离子通道做了简要的总结和展望, 该综述对于发展新型人工离子 通道以及发掘其潜在的应用价值具有重要意义.

关键词 大环分子; 离子识别; 跨膜通道; 结构调控; 生物应用
\end{abstract}

\section{Research Progress on the Macrocycle-Derived Artificial Transmembrane Ion Channels}

\author{
Tengfei Yan ${ }^{a, b} \quad$ Shengda Liu ${ }^{a, b} \quad$ Yichen Luo $^{b} \quad$ Yingping Zou* ${ }^{*, a} \quad$ Junqiu Liu* ${ }^{*, b, c}$ \\ ( ${ }^{a}$ College of Chemistry and Chemical Engineering, Central South University, Changsha 410083, China) \\ ( ${ }^{b}$ College of Material, Chemistry and Chemical Engineering, Hangzhou Normal University, Hangzhou 310036, China) \\ ( ${ }^{c}$ College of Chemistry, State Key Laboratory of Supramolecular Structure and Materials, Jilin University, \\ Changchun 130012, China)
}

\begin{abstract}
Macrocycles such as cyclodextrin and crown ether are applied to construct artificial transmembrane ion transport systems owing to their unique cavity structure and the ability to recognize molecules and ions via host-guest interaction. Compared with natural channel proteins, macrocycles have many advantages, such as the low cost, stabilities, easy structural modification and functionalization, etc., which make them preferable candidates for preparing artificial ion channel. Herein, we reviewed the recent progress of macrocycles-based artificial ion channels, and systematically summarized the preparation methods, structural regulation and potential applications of the artificial ion channels based on different macrocycles. Finally, we have briefly summarized and outlooked the progress of macrocycle-based artificial ion channels. This review is of great significance for developing novel artificial transmembrane ion channels and exploring their potential applications.
\end{abstract}

Keywords macrocycle; ion recognition; transmembrane channel; structural regulation; biological application

\section{1 引言}

在自然界的有机生命体中, 由膜通道蛋白所精确调 控的离子跨膜传输在维持生命体的生理机能方面扮演 着举足轻重的角色, 例如细胞内外渗透压调控、肌肉收 缩、神经信号传输等功能的实现都需要不同种类通道蛋 白的参与 ${ }^{[1-2]}$. 生命体内的通道蛋白发生突变导致其功 能缺失则可能引发各种离子通道疾病(例如囊性纤维 化、心律失常等 $)^{[3-4]}$. 与自然进化的蛋白质大分子相比, 人工合成的离子通道或者载体具有结构简单、性能稳 定、价格低廉等优点. 因此通过仿生的思路, 以天然通 道为模型开发新型的人工离子通道, 对于探索离子通道
的结构和性质关系以及开发其在医药和化工产业中的 潜在应用具有重要的意义 ${ }^{[5-6]}$. 本综述以大环类(如环糊 精、冠醚、杯芳烃、柱芳烃、葫芦艮、环肽以及芳香平 面大环等)人工跨膜离子通道的构筑、结构调控以及生 物医学应用为主线, 针对近年来国内外相关课题组的研 究报道进行总结论述, 并对大环类人工跨膜通道的未来 发展进行展望(图 1).

\section{2 人工离子通道的构筑}

\section{1 环糊精}

环糊精是一类由吡喃葡萄糖单元构成的大环分子,

\footnotetext{
* E-mail: yingpingzou@csu.edu.cn; junqiuliu@jlu.edu.cn

Received May 19, 2021; published June 24, 2021.

Project supported by the National Natural Science Foundation of China (Nos. 21875286, 22001054, 22075065).

项目受国家自然科学基金(Nos. 21875286, 22001054, 22075065)资助.
} 


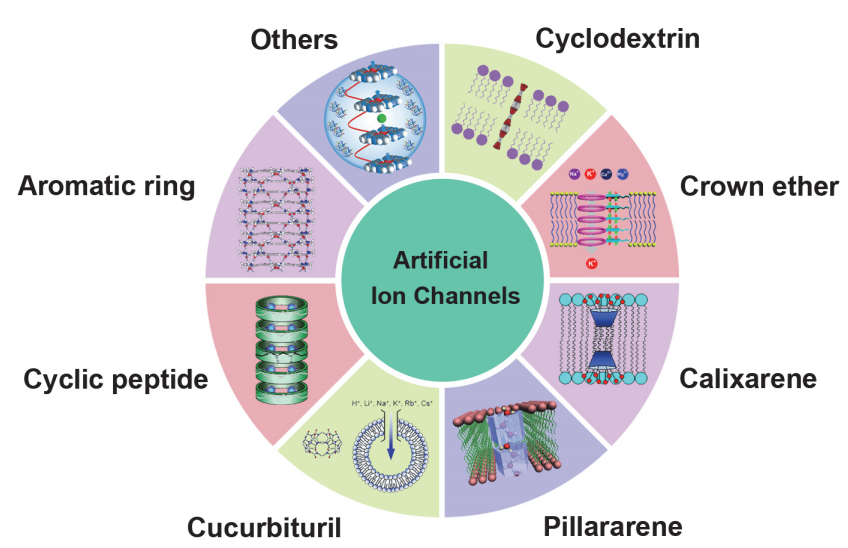

图 1 基于不同种类大环分子的人工跨膜离子通道示意图

Figure 1 The schematic illustration of artificial transmembrane ion channels based on different kind of macrocycles

通常是由淀粉酶对淀粉的水解获得. 环糊精能够通过分 子间弱相互作用与多种小分子、金属离子等形成稳定的 主-客体复合物 ${ }^{[7-9]}$. 第一例人工离子通道被公认为是 Tabushi 等 ${ }^{[10]}$ 在 1982 年所报道的基于 $\beta$-环糊精衍生物的 通道结构, 研究人员将 $\beta$-环糊精的一端修饰上脂溶性的 烷基链以增加通道分子的脂溶性(图 2a, 化合物 1), 由 于该分子的尺寸较短, 所以只能以二聚体的形式在磷脂 膜上形成超分子孔道结构. 脂质体荧光实验表明该通道 分子对于 $\mathrm{Cu}^{2+}$ 和 $\mathrm{Co}^{2+}$ 等金属离子表现出特异性跨膜传 输能力. 然而受限于当时的测试条件, 并不能排除离子 载体的跨膜传输机制, 因此该项研究并没有十分准确地 证明离子通道的存在. 直到 20 世纪 90 年代初, 膜片钳 技术的发展和应用才使得具体量化人工离子通道的各 项传输性质成为可能 ${ }^{[11]}$. 受到 Tabushi 等的启发, Gin 等 ${ }^{[12]}$ 将 $\beta$-环糊精与疏水烷基链通过仲胺基团连接得到 一种新型的孔道结构(图 2a, 化合物 2), 由于该通道分 子内的仲胺基团与阴离子之间存在电荷偶极相互作用, 因此对阴离子 (如 $\mathrm{I}^{-}, \mathrm{Br}^{-}, \mathrm{Cl}^{-}$等) 表现出特异性跨膜传输, 且选择性顺序为 $\mathrm{I}^{-}>\mathrm{Br}^{-}>\mathrm{Cl}^{-}$. 除了通过分子间弱相
互作用形成的超分子离子通道之外，科学家们也尝试构 筑基于环糊精结构的单分子离子跨膜通道. 例如 Pelta 课题组 ${ }^{[13]}$ 曾利用聚乙二醇衍生物作为线性轴分子，通 过 “穿线法” 将多个 $\alpha$-环糊精连接成聚轮烷, 然后利用 共价键将 $\alpha$-环糊精进行交联并将轴分子两侧的大体积 封端基团进行水解，最后得到含有多个 $\alpha$-环糊精基元的 单分子纳米管状结构. 该体系最大的优点是可以通过调 节线性轴分子的长度使其与磷脂膜的厚度 $(5 \mathrm{~nm})$ 相匹 配. 通过平面脂双层电导实验, 得到了 $\mathrm{K}^{+}$在跨膜传输过 程中的方波型电流信号, 且单通道电流强度可以达到 $(6.0 \pm 0.1) \mathrm{pA}$, 充分证实了该结构的离子通道性质(图 $2 b)$.

\section{2 冠醚}

自从 20 世纪 60 年代被 Pederson ${ }^{[14]}$ 发现以来, 冠醚 因其简单的结构和独特的碱金属离子结合能力而被广 泛应用于人工跨膜离子通道或者载体的设计与开 发 ${ }^{[14-15]}$. 在溶液体系中, 冠醚和碱金属离子 $\left(\mathrm{Li}^{+} 、 \mathrm{Na}^{+} 、\right.$

$\left.\mathrm{K}^{+} 、 \mathrm{Rb}^{+} 、 \mathrm{Cs}^{+}\right)$之间的结合基本遵循冠醚环空腔和碱金 属离子直径之间大小匹配关系. 例如 12-冠醚-4 在溶液 中会选择性地结合尺寸较小 $\mathrm{Li}^{+}, 15$ - 冠醚-5 和 18-冠醚-6 则分别选择性结合 $\mathrm{Na}^{+}$和 $\mathrm{K}^{+}$, 而 21-冠醚-7 则对尺寸较 大的 $\mathrm{Rb}^{+}$和 $\mathrm{Cs}^{+}$均有一定的结合能力, 因此冠醚分子常 被用于高选择性的人工跨膜离子传输体系的构筑 ${ }^{[16]}$. Gokel 等 ${ }^{[17]}$ 在 1990 年首次合成了一种三聚冠醚类人工 跨膜离子通道, 将三个 $\mathrm{N}$ 杂冠醚结构利用疏水烷基链连 接形成一种能够横跨磷脂双层膜的单分子通道模型(图 $2 \mathrm{c}$, 化合物 3). 溶液中的阳离子首先通过端基的冠醚分 子进行复合, 然后借助中间的冠醚进行跳跃并最终实现 跨膜转运, 测试结果表明, 该通道对 $\mathrm{Na}^{+}$的传输速率可 以达到 $13.5 \mathrm{~s}^{-1}$, 尽管远低于短杆菌肽 $\mathrm{A}$ 的传输速率 (1.4 $\times 10^{3} \mathrm{~s}^{-1}$ ), 但是这一简单的人工通道模型具备天然通道 完备的结构和功能特征, 仍然是人工跨膜通道领域早期 最经典的分子之一. 随后该课题组 ${ }^{18-19]}$ 还发现这种三聚 (a)

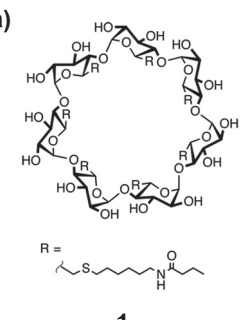

1

(b)

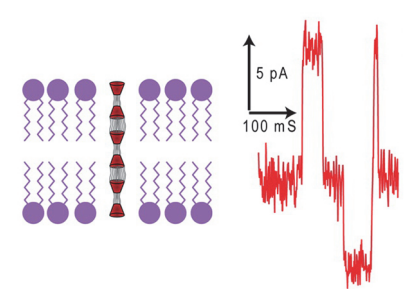

(c)

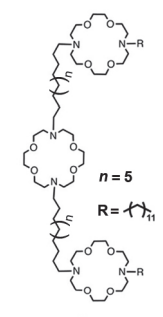

3

(d)

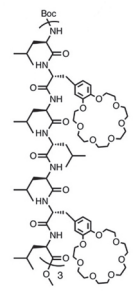

4

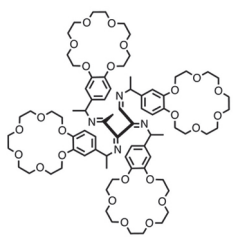

(e)

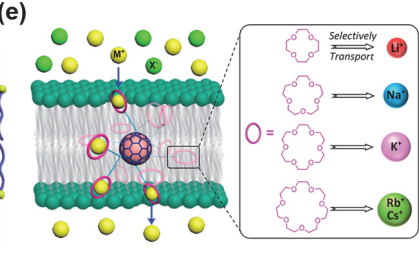

图 2 基于 $(a, b)$ 环糊精以及 $(\mathrm{c} \sim \mathrm{e})$ 冠醚结构的人工跨膜离子通道

Figure 2 Artificial transmembrane ion channels based on $(\mathrm{a}, \mathrm{b})$ cyclodextrins and $(\mathrm{c} \sim \mathrm{e})$ crown ethers 
冠醚类通道分子还对革兰氏阳性菌和革兰氏阴性菌都 具有明显的抑菌活性. 受到 Gokel 等研究工作的启发, Nolte ${ }^{[20]}$ 和 Voyer ${ }^{[21]}$ 等课题组通过以线性聚合物、多肽为 骨架，并接枝多个冠醚基团使其排列成规则的孔道结 构, 同样实现了对一价碱金属离子的跨膜转运(图 2c, 化合物 4 和 5). 近年来, Zeng 等 ${ }^{[22]}$ 利用冠醚对碱金属离 子的特异选择性, 发展了一系列功能性人工跨膜离子传 输体系. 2017 年, 该课题组使用了一个能够形成稳定的 一维氢键结构的烷基链作为 18-冠醚-6 和 15-冠醚-5 的 侧链, 并通过超分子自组装的方式使其在磷脂双层膜中 形成稳定孔道结构. 通过对侧链长度的调节, 实现了通 道结构对 $\mathrm{K}^{+}$的选择性高效跨膜传递, 其对离子传输的 $\mathrm{K}^{+} / \mathrm{Na}^{+}$选择性和半数有效浓度 $\left(\mathrm{EC}_{50}\right)$ 分别 9.8 和 6.2 $\mu \mathrm{mol} \cdot \mathrm{L}^{-1}$ (图 $2 \mathrm{~d}$ ). 随后, Zeng 等 ${ }^{[23]}$ 又利用 $\mathrm{C}_{60}$-富勒烯作 为核心, 通过长度可调的柔性烷基链与外围的十二个苯 并冠醚基团相连, 从而形成一个直径和磷脂双分子层厚 度相近的球形三维结构. 通过改变 $\mathrm{C}_{60}$ 外侧相连的冠醚 类型，成功实现了对不同碱金属离子的高效且选择性跨 膜传输, 例如当 $\mathrm{C}_{60}$ 外侧连接 12-冠醚-4 或 21-冠醚-7 时, 该结构对 $\mathrm{Li}^{+}, \mathrm{Rb}^{+}, \mathrm{Cs}^{+}$的离子传输 $\mathrm{EC}_{50}$ 分别为 0.13 $\mu \mathrm{mol} \cdot \mathrm{L}^{-1}, 105 \mathrm{nmol} \cdot \mathrm{L}^{-1}, 77 \mathrm{nmol} \cdot \mathrm{L}^{-1}$. 而当 $\mathrm{C}_{60}$ 外侧连接 15 -冠醚-5 或 18-冠醚-6 时, 其离子跨膜传输的 $\mathrm{Na}^{+} / \mathrm{K}^{+}$和 $\mathrm{K}^{+} / \mathrm{Na}^{+}$选择性最高分别可以达到 13.7 和 7.8(图 2e). 除碱 金属离子通道之外, Zeng 等还构筑了一系列其它类型通

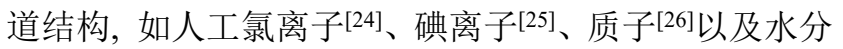
子 [27]通道等, 为新型人工跨膜通道的构筑提供了许多 新的思路和策略.

\section{3 杯芳烃}

杯芳烃被认为是继冠醚和环糊精之后的第三代主 体大环分子, 由若干个苯酚单元通过亚甲基(或杂原子, 如氧、硫、氮等)在酚羟基邻位相连接而形成的环状低 聚物 ${ }^{[28]}$. 因其结构像一个酒杯而被称为杯芳烃. 杯芳烃 具有拥有特殊的结构且构象稳定便于调控以及上下缘 都易于衍生化等特点, 使得杯芳烃在分子、离子识别等 领域有着广泛的研究 ${ }^{[29-30]} .1995$ 年, Kobuke 课题组 ${ }^{[31]}$ 首 次报道了一种基于杯 [4]芳烃的非肽类人工钾离子通道, 利用间苯二酚与十八烷醛缩合得到一种两亲性杯 [4]间 苯二酚芳烃衍生物. 该结构在插入平面脂双层后, 由四 个间苯二酚环形成的空腔经过四条烷基链延伸以尾对 尾形式在磷脂膜上形成二聚体形式的孔道结构(图 3a, 化合物 6). 该通道的离子选择性由杯[4]间苯二酚芳烃 结构中最窄的区域所决定, 4 个酪氨酸残基所形成的“芳 香笼” 可以通过较弱的静电作用, 促使 $\mathrm{K}^{+}$完全脱水进行 跨膜转运. 平面脂双层电导实验表明, 6 对 $\mathrm{K}^{+}$的电导率 可以达到(6.1 \pm 0.8$) \mathrm{pS}$. 对于尺寸较大的金属离子如 $\mathrm{Rb}^{+} 、 \mathrm{Cs}^{+}$以及 $\mathrm{Cl}^{-}$等并没有表现出明显的传输活性 ${ }^{[31]}$. 随 后, 研究人员又利用胆酸作为尾部基团替代烷基链来优 化离子传递路径(图 3a, 化合物 7), 可以有效地提高离
子选择性, 并且能够使离子通道在磷脂膜上保持长时间 的开放状态 ${ }^{[32]}$. 如图 3b, 3c 所示, 由化合物 7 和 6 组成 的离子通道的打开和关闭状态时间比值约为 95 比 5 , 其 中通道分子 7 的平均开放时间为 $2.5 \sim 4.5 \mathrm{~s}$, 而 6 的嵌膜 稳定性较弱, 其通道平均开放时间仅为 $(35 \pm 10) \mathrm{ms}$.

(a)
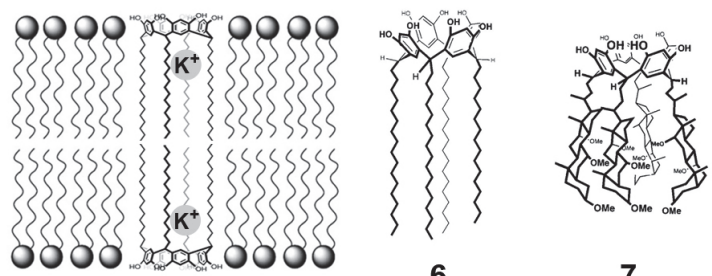

6

7

(b)

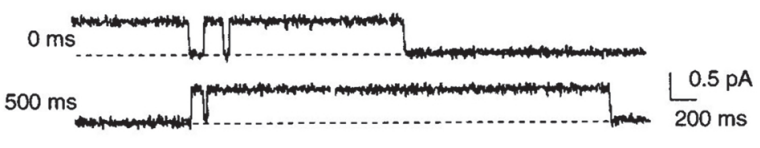

(c)

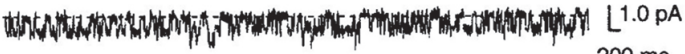

$200 \mathrm{~ms}$

图 3 (a)基于杯 [4]芳烃的人工跨膜离子通道; 化合物(b) 7 和(c) 6 的平 面脂双层电流信号图

Figure 3 (a) Artificial transmembrane ion channels based on calix[4]arene; electrophysiological signals of the compounds (b) 7 and (c) 6 recorded by planer lipid bilayer

\section{4 柱芳烃}

2008 年, Ogoshi 和 Nakamoto 等 ${ }^{[33]}$ 首次报道了一类 由对苯二酚通过亚甲基桥联的柱状大环分子即柱[5]芳 烃的合成. 由于柱芳烃具有独特的对称结构和卓越的分 子识别能力, 因而格外受到科研工作者们的关注 ${ }^{[34-35]}$. 2011 年, Hou 等 ${ }^{[36]}$ 对全酯化柱 [5]芳烃的单晶结构分析时 发现其内部包裹一条规则水线序列, 通过将其中单晶浸 泡在同位素标记的水中进行测试，首次发现柱[5]芳烃 晶体的质子传导性质. 由于单个柱[5]芳烃分子较短与 磷脂膜的厚度不匹配, 因此利用不同长度的烷基链使两 个柱[5]芳烃相连接, 使其能够在磷脂膜中形成稳定的 单分子通道结构. 溶液中的质子通过柱芳烃内部规则排 列的水线结构进行快速 “跳跃式” 传输(图 4a). 研究表 明该通道质子传输的电导率可以达到 $6 \mathrm{pS}$, 可以与天然 M2 质子通道的传输活性相謧美, 在人工跨膜通道体系 中，该工作是第一例基于水线传输机制进行质子跨膜传 递的人工离子通道 ${ }^{[37]}$. 此外, 研究人员发现在柱[5]芳烃 两侧引入负电荷的基团, 不仅可以显著增强其嵌膜能力 还能够利用电荷与离子之间相互作用, 实现对不同离子 的选择性跨膜传输. 例如在柱 [5]芳烃两侧引入含有磷 酸二氢根的短肽侧链不仅可以使通道分子长度达到 3.5 $\mathrm{nm}$ 以保证单分子嵌膜, 而且还能使通道分子可以在极 低浓度下实现对 $\mathrm{NH}_{4}{ }^{+}$选择性跨膜传递, 且对不同离子 选择性传输顺序为 $\mathrm{NH}_{4}^{+}>\mathrm{Cs}^{+}>\mathrm{Rb}^{+}>\mathrm{K}^{+}>\mathrm{Cl}^{-}>\mathrm{Na}^{+}$. 在该体系中磷酸二氢根基团和 $\mathrm{NH}_{4}{ }^{+}$之间的较强的静电 
吸引以及氢键相互作用是实现 $\mathrm{NH}_{4}{ }^{+}$高选择性传输的关 键 ${ }^{[38]}$.

(a)

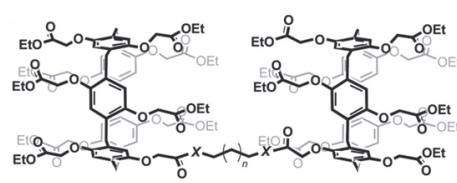

8

(b)

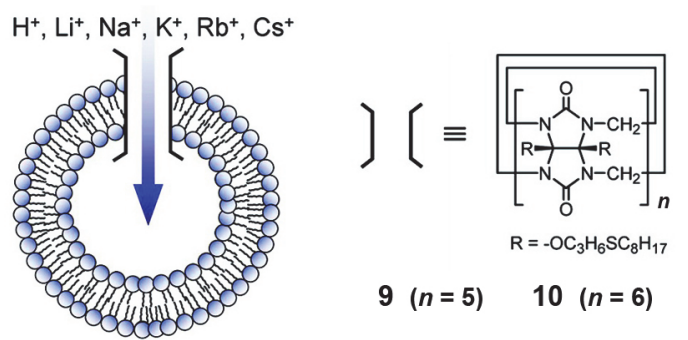

图 4 基于(a)柱 [5]芳烃以及(b)葫芦 [5]/[6]腿的人工跨膜离子通道

Figure 4 Artificial transmembrane ion channels based on (a) pillar[5]arene and (b) cucurbit[5]/[6]urils

\section{5 葫芦艮}

葫芦嫝是一类甘嫝单元和桥连亚甲基组成的大环 结构, 由于该化合物的形状近似于葫芦科植物南瓜, 因 此将其命名为葫芦脲 ${ }^{[39]}$. 相比于上述几类大环分子, 关 于葫芦嫝的人工离子通道的研究相对较少, 主要因为葫 芦脲分子价格相对较为昂贵且不易进行修饰. 目前只有 Kim 等 ${ }^{[40-41]}$ 少数课题组对葫芦脲的修饰和功能化有较 为深入的研究. 受到天然钾离子通道通过尺寸效应精准 实现对 $\mathrm{K}^{+}$的跨膜传递行为的启发 ${ }^{[42]} .2004$ 年, Kim 等 ${ }^{[43]}$ 报道了一类含有脂溶性烷基侧链的葫芦 [5]/[6]䐖人工通 道分子(图 4b, 化合物 9 和 10), 葫芦 [6]嫝的疏水内腔直 径约为 $0.55 \mathrm{~nm}$, 而其两端因为含有较多指向轴心的羰
基，所以其两端的端口直径较窄约为 $0.39 \mathrm{~nm}$. 因此利 用类似于天然钾离子通道蛋白尺寸效应，通道分子 $\mathbf{1 0}$ 实现了对一价碱金属离子 (选择性顺序: $\mathrm{Li}^{+}>\mathrm{Cs}^{+} \approx \mathrm{Rb}^{+}$ $>\mathrm{K}^{+}>\mathrm{Na}^{+}$) 的跨膜传输. 平面脂双层电导实验表明, 在 $80 \mathrm{mV}$ 电压的驱动下, 单分子通道 $\mathbf{1 0}$ 对 $\mathrm{Cs}^{+}$的跨膜传递 速率高达 $3 \times 10^{7}$ 个/秒. 此外, 由于乙酰胆碱能够与葫芦 [6]嫝发生主-客体复合 $\left(k \approx 10^{3}\right)$, 因此利用乙酰胆碱作为 客体分子还可以实现对离子传输性质的可控调节. 与 10 相似，基于葫芦[5]嫝的通道分子 9 同样表现出了相似 的离子跨膜传递性质(图 4b).

\section{6 环形多肽}

天然的多肽分子如短杆菌肽 $\mathrm{A}$ 能够以二聚体的形 式横贯细胞膜, 实现对质子、一价碱金属离子以及水分 子的跨膜传输 ${ }^{[44]}$. 如何通过向自然学习, 设计并合成与 天然结构类似的人工跨膜传输系统仍然是一个艰巨的 任务 ${ }^{[45]} .1994$ 年, Ghadiri 等 ${ }^{[46]}$ 提出了一种基于环肽分子 自组装形成圆柱状人工跨膜离子通道的设计策略. 利用 $L$-色氨酸和 $D$-亮氨酸残基交替连接形成一种内径约为 $0.75 \mathrm{~nm}$ 的八元环形多肽(图 5a, 化合物 11), 多肽结构良 好的脂溶性以及其分子间的氢键相互作用保证其在磷 脂膜内能够形成规则孔道结构(图 5b). 这种基于环形多 肽的离子通道在极低的浓度下 $\left(10^{-7} \mathrm{~mol} \cdot \mathrm{L}^{-1}\right)$ 仍然具有 惊人的离子传输活性, 其对 $\mathrm{Na}^{+} 、 \mathrm{~K}^{+}$等碱金属离子的跨 膜传输速率均高于 $10^{7}$ 个/秒, 其离子传输速率可以和 短杆菌肽 A 以及两性霉素 B 相謧美. 该方法最大的优点 是能够通过改变环肽亚基氨基酸组成，实现对环肽纳米 管内径和外表面性质的可控调节. 由于具有较好的嵌膜 性质以及超高的离子传输活性，基于环肽堆积所形成的 离子通道的开发对于设计新型细胞毒性药物、离子转运 体以及药物载体等具有重要的意义 ${ }^{[47]}$. (a)

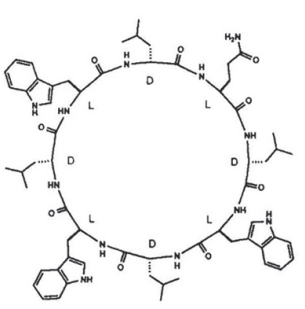

11

(b)

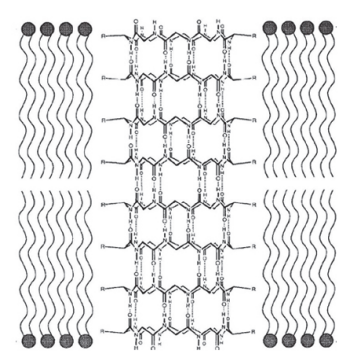

(c)

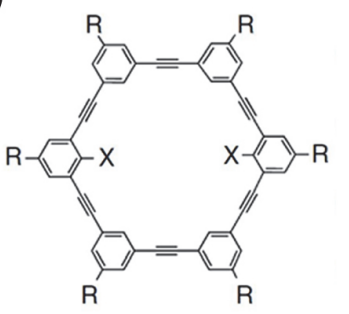

$12 \mathrm{R}=\mathrm{N}_{\mathrm{H}} \mathrm{C}_{\mathrm{O}}^{\mathrm{O}} \mathrm{O}^{-} \mathrm{C}_{4} \mathrm{H}_{9} \quad \mathrm{X}=\mathrm{H}$

$13 \mathrm{R}=\mathrm{N}_{\mathrm{H}} \mathrm{T}_{\mathrm{O}}^{\mathrm{O}} \mathrm{O}_{n-\mathrm{C}_{8} \mathrm{H}_{17}} \mathrm{X}=\mathrm{H}$

$14 \mathrm{R}=\widehat{H}_{\mathrm{H}} \mathrm{C}_{\mathrm{O}}^{\mathrm{O}} \cdot \mathrm{C}_{4} \mathrm{H}_{9} \quad \mathrm{X}=\mathrm{CH}_{3}$

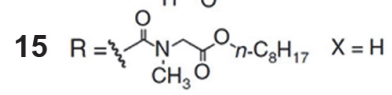

(d)

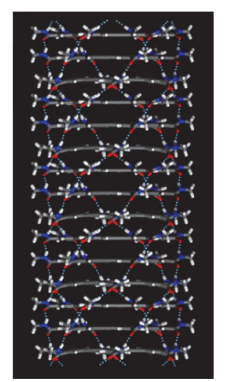

(e)

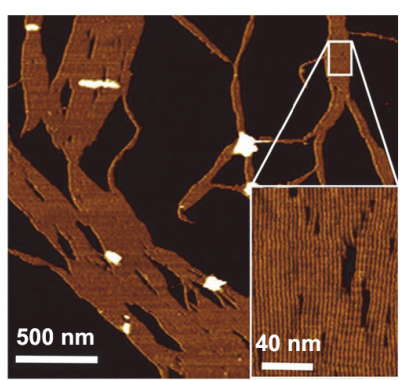

图 5 基于 $(\mathrm{a}, \mathrm{b})$ 环形多肽以及 $(\mathrm{c} \sim \mathrm{e})$ 刚性平面芳香大环结构的人工跨膜离子通道

Figure 5 Artificial transmembrane ion channels based on $(a, b)$ cyclic peptide and $(c \sim e)$ rigid flat aromatic ring 


\section{7 芳香平面大环}

与环肽通过分子间堆积所形成的孔道结构类似, 平 面的芳香大环结构也可以通过分子间的 $\pi-\pi$ 相互作用组 装成超分子通道. 例如 Gong 等 ${ }^{[48]}$ 利用六(间苯乙炔撑) 为内核, 以酰胺键连接的烷基链作为脂溶性基团, 构筑 了一系列刚性平面大环分子 $12 \sim 15$ (图 5c). 化合物 12 可以在磷脂膜中自发堆积形成孔道结构(图 5d), 能够实 现对质子、阴离子以及水分子的高效跨膜传输, 其对 $\mathrm{H}^{+} / \mathrm{Cl}^{-}$和 $\mathrm{H}^{+} / \mathrm{K}^{+}$的传输选择性分别约为 3000 和 2000 . 通 过希尔分析计算该大环通道离子传输的希尔系数为 $9 \pm$ 3 , 说明该离子通道平均由 9 个大环单体分子堆积而成. 此外, 通道的传输活性受内部结构影响很大, 大环内部 含有两个甲基的 14 则没有表现出明显的通道活性. 作 为对照分子, 15 上的 $\mathrm{N}$ 原子被甲基化, 不能与相邻大环 形成稳定氢键网络, 因此也不具备离子传输活性. 圆二 色谱表明 13 的分子间堆积能力较弱, 不能在磷脂膜中 形成稳定的孔道结构. 此外, 研究人员还利用原子力显 微镜直接地观测到大环分子的堆积形貌图, 较为直观地 反映出这种大环结构较强的堆积能力(图 5e).

\section{8 其他}

除上述几种常见的大环分子之外, 还有一些特殊的 大环结构 G-四联体、金属-有机化合物等也曾被用于人 工跨膜离子通道的构筑. 关于 $\mathrm{G}$-四联体的研究兴起于 20 世纪 90 年代 ${ }^{[49]}$, 它是一种富含鸟嘌呤碱基的特殊四 链核酸结构, 通过 Hoogsteen 氢键进行碱基配对形成平 面正方形的四聚体结构, 两个或多个鸟嘌呤四聚体在一 价阳离子(如 $\mathrm{Na}^{+} 、 \mathrm{~K}^{+}$或 $\mathrm{NH}_{4}^{+}$等) 的配位作用下，通过分 子间的 $\pi-\pi$ 共轭形成稳定的 $\mathrm{G}$-四联体二级结构 ${ }^{[50]} .2006$ 年, Davis 等 ${ }^{[51]}$ 将烯丙醚基团引入到鸟嘌呤单体, 随后利 用钾离子诱导单体自组装并利用烯烃复分解反应对双 键进行交联得到长度约为 $3.0 \mathrm{~nm}$ 的稳定 $\mathrm{G}$-四联体二级

(a)
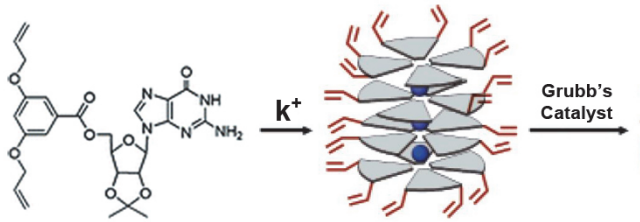

16

(c)

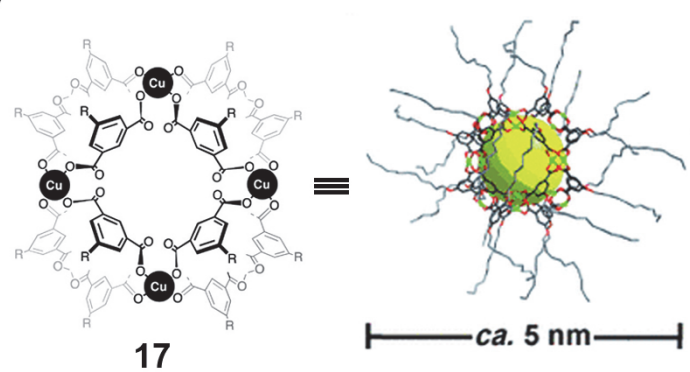

结构(图 6a，6b). 脂质体苂光实验以及核磁钠谱分析表 明，发现该结构对溶液中的 $\mathrm{Na}^{+}$表现出明显的跨膜传输 活性. 此外, 三维的金属-有机多面体(MOP)也可用于构 建离子跨膜传输体系, 2006 年 $\mathrm{Kim}$ 研究组 ${ }^{[52]}$ 利用 MOP-18 构建了一种新型的人工离子通道(图 6c, 6d), 其 分子式为 $\mathrm{Cu}_{24}\left(5-\mathrm{OC}_{12} \mathrm{H}_{25}-\mathrm{mBDC}\right)_{24}, \mathrm{X}$-射线单晶衍射数 据表明 MOP-18 的直径约为 $5 \mathrm{~nm}$ 与磷脂双层膜的厚度 相匹配, 且 MOP-18 分子中间有一个内径约为 $1.38 \mathrm{~nm}$ 的空腔结构可以作为离子跨膜传递的通路. 脂质体苂光 实验表明 MOP-18 能够实现对质子以及一价碱金属离子 的跨膜传递, 且传输顺序为 $\mathrm{Li}^{+}>\mathrm{Na}^{+}>\mathrm{K}^{+}>\mathrm{Rb}^{+}>\mathrm{Cs}^{+}$, 其离子传输选择性符合 Eisenman XI 传输机制[53]. 科学 家们也尝试通过将不同大环分子进行结合来构建杂合 类型的孔道结构, 例如 Xin 等 ${ }^{[54]}$ 利用点击化学反应将叠 氮化的 $\alpha$-环糊精修饰在炔基柱 [5]芳烃的两端，形成一 种中间疏水两端亲水的环糊精-柱芳烃杂合孔道. 这种 三嵌段结构与磷脂膜具有相似的亲-疏水性质, 因此具 有优异的嵌膜能力. 通过调节环糊精和柱芳烃之间烷基 链的长度, 实现了对碱金属离子的高效传输, 并且该杂 合通道还表现出较高的 $\mathrm{K}^{+} / \mathrm{Na}^{+}$选择性. 这些发现为天然 $\mathrm{K}^{+}$通道提供了仿生替代品, 并可能在离子分离以及医 药开发中发现潜在应用.

\section{3 人工离子通道的结构调控}

\section{1 开-关性质调控}

天然离子通道对外界刺激往往具有可控、高效的传 输特点. 受到大自然的启发, 科研工作者利用化学合成 的方法同样制备了各种各样的门控离子通道 ${ }^{[55]}$. 2015 年, Bao 等[56]利用光敏性的邻硝基苠酯基团将苯并 18冠醚-6 与脂溶性烷基链相连接形成一种双亲分子(图 7a, 化合物 18). 该结构能够在磷脂双层膜中自发堆积形成

(b)
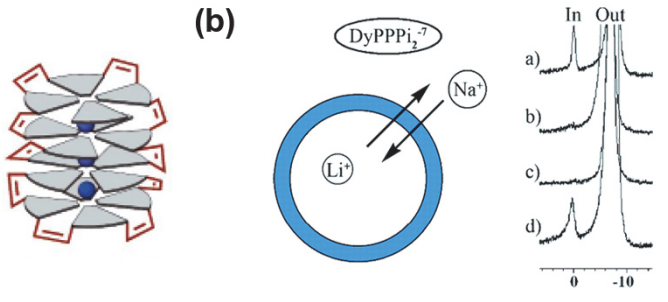

$\delta$

(d)

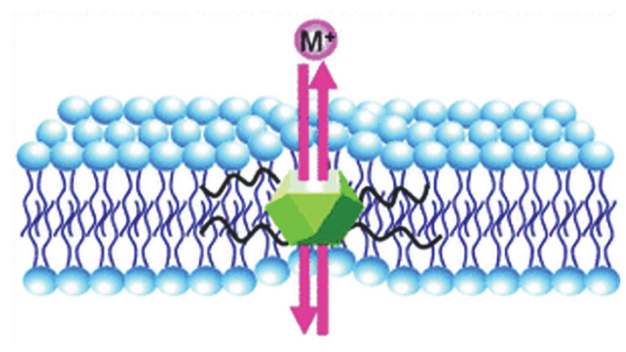

图 6 基于 $(a, b)$ G-四联体以及 $(c, d)$ 金属-有机多面体结构的人工跨膜离子通道

Figure 6 Artificial transmembrane ion channels based on (a, b) G-quartets and (c, d) metal-organic polyhedra 
(a)
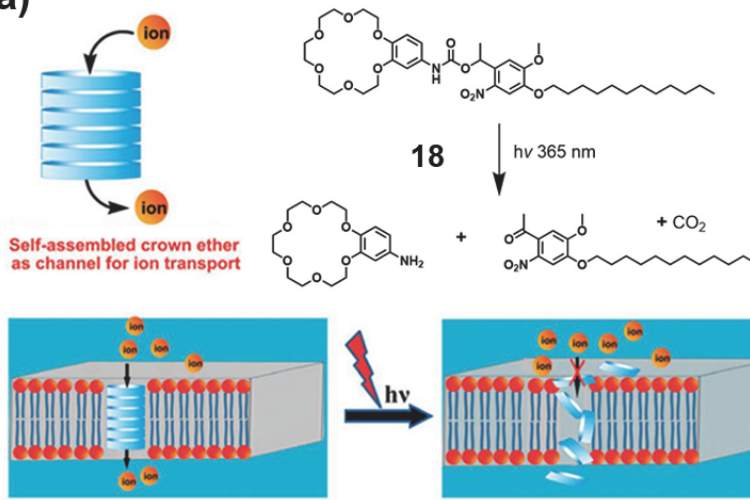
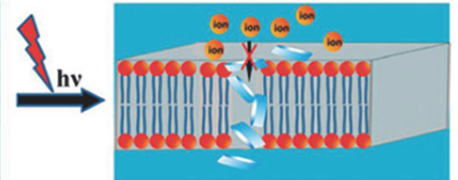

(b)

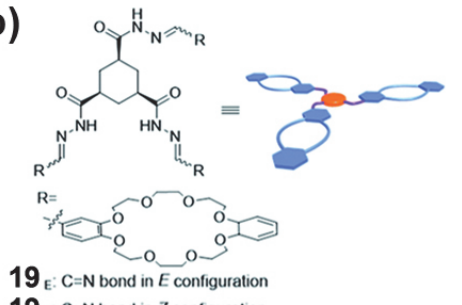

19. $\mathrm{C}=\mathrm{N}$ bond in $Z$ configuration

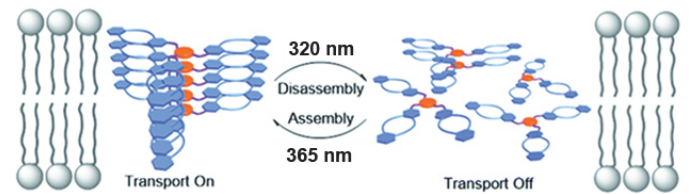

图 7 具有刺激响应功能的智能人工跨膜离子通道

Figure 7 Intelligent artificial transmembrane ion channels with stimuli-responsive abilities

超分子孔道结构, 并对 $\mathrm{K}^{+}$表现出一定的选择性跨膜传 输. 当通道结构受到 $365 \mathrm{~nm}$ 的紫外光照射后, 其双亲结 构被破坏从而引起通道的离子传输性质的下降. 且随着 光照时间的增长通道传输性质逐渐减弱至基本消失. 脂 质体苂光实验和膜片钳单通道电流实验都证实了该离 子通道的光敏特性(图 7a). 尽管没有实现对离子通道 开-关的可逆调控, 但该体系仍然为新型智能人工离子 通道的开发提供了一种新思路. 随后, Liu 等 ${ }^{[57]}$ 报道了 一种通过酰腙键连接 24 -冠醚- 8 的三联体分子. 在溶液 态下, 该三联体结构可以通过分子间的氢键作用自组装 形成超分子孔道结构, 并对 $\mathrm{NH}_{4}{ }^{+}$表现出选择性跨膜转 运. 有趣的是, 利用 $320 \mathrm{~nm}$ 和 $365 \mathrm{~nm}$ 的紫外光对三联 体进行照射可以实现对酰腙键上 $\mathrm{C}=\mathrm{N}$ 键的 $E$ 和 $Z$ 构象 的可逆调控, 从而实现对离子孔道结构的解组装与再组 装的调控并最终实现对离子通道开-关的可逆调节(图 7b). 钾离子通道蛋白是一类重要的膜蛋白, 它在跨膜 输送钾离子的过程中展现出了很好的电压门控特性以 及输送方向性 ${ }^{[58]}$. 如何利用人工合成的体系来模拟天 然的钾离子通道蛋白的电压门控特性一直是科学家们 追逐的目标. 2014 年, Hou 等 ${ }^{[59}$ 通过在柱[5]芳烃两侧引 入带正电荷精氨酸多肽的方式, 构建了一种新型的仿生 智能具有电压门控特性的钾离子通道. 当向磷脂膜两侧 施加一定电压后, 可以驱动带有正电荷的柱[5]芳烃通 道嵌入磷脂内部并且能够稳定地进行钾离子的跨膜传 输. 相反, 当对体系施加一个更高的反转电压时, 由于 通道分子与磷脂膜间的相互作用被破坏, 导致其发生解 离, 从而停止对钾离子的跨膜传输. 众所周知, 具有代 谢活性的细菌的细胞膜表面通常是带负电荷, 因此对带 正电荷的阳离子具有较高的亲和能力. 研究人员对该电 压门控通道的抗菌活性进行了深入研究. 结果表明, 该 离子通道对革兰氏阳性枯草杆菌表现出明显的抗菌活 性, 其半抑制浓度 $\left(\mathrm{IC}_{50}\right)$ 约为 $10 \mu \mathrm{mol} \cdot \mathrm{L}^{-1}$ 与天然多肽丙 甲菌素的抗菌活性达到同一数量级. 该研究结果对于理 解天然离子通道的行为机制具有重要的生物学意义, 也 为开发在生物学和医药学领域具有潜在应用的智能仿

生纳米通道提供了全新的思路.

\section{2 传输性质调控}

除了模拟天然通道蛋白的开-关特性以外, 科学家 们还尝试对人工离子通道的传输选择性进行调控. 2008 年, Gin 课题组 ${ }^{[60]}$ 通过将光敏性的偶氮苯基团共价修饰 到 $\beta$-环糊精的一端, 并利用紫外和可见光的循环照射, 实现了对阴、阳离子传输活性的可逆光控调节. 如图 8a 所示，当偶氮苯为反式构象时，可以通过分子间的主客体相互作用与 $\beta$-环糊精的疏水空腔结合, 此时, 体积 较小的阳离子可以实现高选择性跨膜传输. 当通道结构 受到 $365 \mathrm{~nm}$ 的紫外光照射后, 偶氮苯基团转变为能量 较高的顺式构象, 并与环糊精主体分子发生解复合, 由 于环糊精末端的仲胺基团对阴离子有较好的电荷偶极 相互作用, 此时通道则表现出对阴离子的选择性传输. Xin 等 ${ }^{[61]}$ 报道了一种对 $\mathrm{pH}$ 敏感的芳香酰肼大环结构

(a)

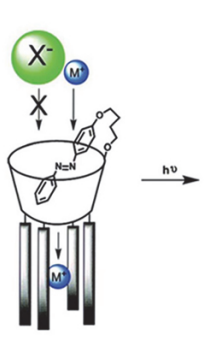

(c)

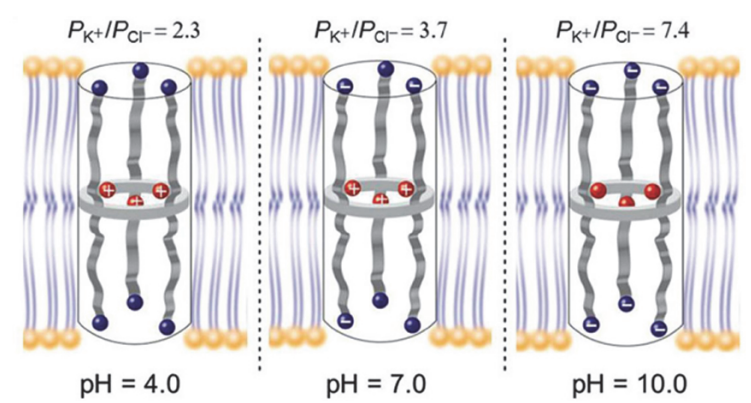

图 8 传输选择性可控的人工跨膜离子通道

Figure 8 Artificial transmembrane ion channels with controllable transport selectivity 
(图 8b). 大环分子的刚性平面结构以及其外侧的苯丙氨 酸三肽侧链保证其能够在磷脂膜中形成稳定的超分子 孔道. 在碱性条件下 $(\mathrm{pH}=10.0)$, 通道分子两端为带有 负电荷的羧酸根基团, 通过正负电荷之间的相互吸引作 用有效促进 $\mathrm{K}^{+}$的跨膜传输, 对 $\mathrm{K}^{+} / \mathrm{Cl}^{-}$的传输选择性可以 达到 7.4. 在酸性条件下 $(\mathrm{pH}=4.0)$, 酰肼大环分子内部 的伯胺基团发生质子化并带有正电荷, 此时通道结构更 有利于 $\mathrm{Cl}^{-}$通过, 此时的 $\mathrm{K}^{+} / \mathrm{Cl}^{-}$的传输选择性降低至 2.3(图 8c). 尽管该体系并没有完全实现通道对正、负电 荷传输的反转的调控, 但是该工作对于构筑新型可控离 子跨膜通道仍然具有重要的借鉴意义.

\section{4 人工离子通道在生物医药领域中的应用}

\section{1 离子通道疾病}

细胞膜上的离子通道蛋白对于维持正常的新陈代 谢和生理功能起着十分重要的作用. 当通道蛋白的结构 或者功能发生异常时, 则会导致生理功能紊乱并引发致 命的离子通道疾病. 常见的离子通道疾病有囊性纤维

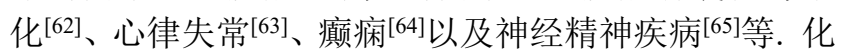
学家们致力于通过有机合成的方法, 构筑与天然通道蛋 白具有相似功能的人工跨膜通道, 为治疗离子通道疾病 提供新的模型和思路. 2019年, Burke 课题组 ${ }^{[66]}$ 报道了一 种利用两性霉素 B 治疗囊性纤维化疾病的新策略. 众所 周知, 囊性纤维化是由呼吸道表皮细胞氯离子通道蛋白 功能缺陷所诱发的一种离子通道疾病. 患者呼吸道会积 累大量酸性粘液, 不仅堵塞肺部使得呼吸困难, 还会让 肺部更容易受到细菌感染. Burke 等没有采用基因治疗 的复杂策略, 反而利用一种小分子替代物(两性霉素 B) 来弥补缺陷蛋白的通道功能. 研究人员利用囊性纤维化 患者捐赠的肺部组织细胞以及患有囊性纤维化的猪为 模型进行研究. 结果表明, 两性霉素 $\mathrm{B}$ 可以明显恢复肺 部细胞的生理功能, 例如 $\mathrm{pH}$ 水平的提高、细胞粘性改 善以及抗菌活性增加等. 研究者表示, 尽管两性霉素 $\mathrm{B}$ 已经(作为抗菌药物)获批上市, 但是在这种药物安全用 于治疗囊性纤维化患者之前, 还需要进行更多的研究来 证实.

\section{2 抗菌、抗癌应用}

除了用于治疗离子通道疾病外, 人工离子通道在抗 菌和抗癌领域同样具有潜在的应用价值. 因为离子通道 在嵌膜之后, 能够破坏细胞膜的完整性, 导致胞内大量 无机盐等内容物外流, 破坏细胞内稳态导致细胞死亡.

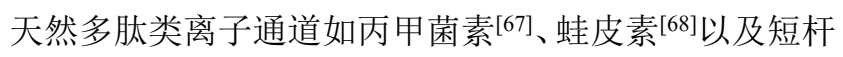
菌肽 ${ }^{[69]}$ 等都具有离子跨膜传输以及抑菌活性. 受到天 然离子通道抗菌性能的启发, 2001 年, Gokel 等 ${ }^{[70]}$ 首次将 三聚冠醚类人工跨膜通道应用于新型抗菌材料的构筑, 并且通过改变连接冠醚基团烷基链的长度使其能够匹 配磷脂膜的厚度从而增强其抑菌活性. 如图 9a 所示, 由 八碳烷基链连接的 21 对革兰氏阴性大肠杆菌的 $\mathrm{IC}_{50}$ 值

为 $1.3 \times 10^{-4} \mathrm{~mol} \cdot \mathrm{L}^{-1}$, 而由十二碳烷基链连接的通道分 子 22 对大肠杆菌的 $\mathrm{IC}_{50}$ 明显降低至 $1.01 \times 10^{-5} \mathrm{~mol} \cdot \mathrm{L}^{-1}$. 研究人员还发现冠醚类人工离子通道除了本身固有的 抗菌活性之外，还可以通过协同作用显著增强抗生素药 物, 例如利福平、红霉素等的抑菌效果(图 $9 b, 9 c)^{[18]}$. 2017 年, Xin 等 ${ }^{[71]}$ 通过将含有不同个数氨基酸残基的短 杆菌肽 A 与柱[5]芳烃相结合的方式, 构筑出一系列不 同长度的三嵌段人工离子通道(图 9d, 化合物 $23 \sim 27$ ). 研究表明，这种三嵌段孔道结构除了对质子以及碱金属 离子表现出传输活性之外，还对革兰氏阳性的枯草芽孢 杆菌、金黄色葡萄球菌以及表皮葡萄球菌等具有明显的 抑菌活性，其抗菌的 $\mathrm{EC}_{50}$ 值(顺序：23>24 $>\mathbf{2 5}>\mathbf{2 6}>$ 27)随着通道分子长度增加而减小, 说明最长的通道分 子 27 具有最高的抗菌活性. 相比于单独的短杆菌肽 $\mathrm{A}$, 这种三嵌段结构的通道分子还具有很低的溶血毒性, 即 便在较高浓度下 $\left(100 \mu \mathrm{mol} \cdot \mathrm{L}^{-1}\right)$ 时, 27 的溶血毒性仍然低 于 $5.5 \%$. 研究人员分析主要是因为柱芳烃的引入使整 个通道分子的长度大于红细胞磷脂膜的厚度 $(\approx 2.5 \mathrm{~nm})$, 降低了其在磷脂双层膜中的稳定性，从而使这种三嵌段 通道结构具有较低的溶血毒性.

(a)

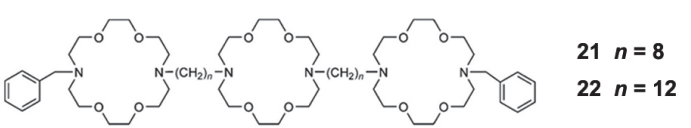

(b)

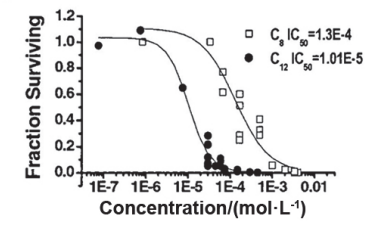

(d)

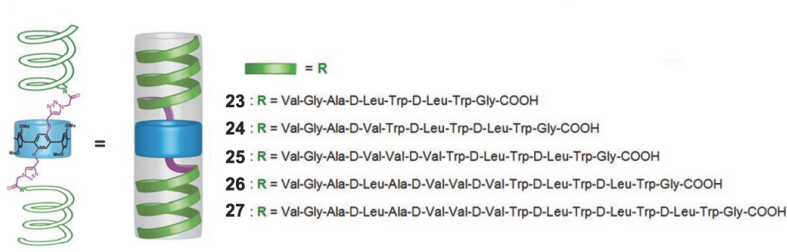

图 9 具有抗菌活性的人工跨膜离子通道

Figure 9 Artificial transmembrane ion channels with antimicrobial activity

癌细胞是一类分裂增殖能力异常强大，几乎可以无 限增殖传代而不启动 “程序性死亡” 过程的异常细 胞 ${ }^{[72-73]}$. 许多针对癌细胞离子通道的研究表明, 因突变 而导致的离子通道功能异常与癌症的发生有密切联系. 例如在乳腺癌、黑色素瘤、前列腺癌等多种癌细胞中都 观察到了钾离子通道的高表达. 因此通过阻断 $\mathrm{K}^{+}$通道 或者阻滞其离子交换过程，来实现对癌细胞增殖的抑制 是抗癌研究中的一个新热点 ${ }^{[74]}$. 此外, $\mathrm{Cl}^{-}$通道在癌细胞 中同样存在异常的高表达现象，并且与肿瘤的大小、远 处转移以及病理分期具有密切的关系. 因此 $\mathrm{Cl}^{-}$通道的 表达水平是癌症诊断中一个有价值的预后指标 ${ }^{[75]}$. 由 
于癌细胞生长迅速, 在代谢的过程中产生大量的酸性代 谢产物, 癌细胞通常会加强 $\mathrm{H}^{+}$通道的功能, 将细胞内多 余的 $\mathrm{H}^{+}$洜出细胞外, 维持细胞内正常生理环境 ${ }^{[76]}$. 目前 临床上已经提出了通过抑制 $\mathrm{H}^{+}$通道活性来杀灭癌细胞 并抑制癌细胞转移的治疗思路. 不过这类研究比较少, 尚且需要更多的试验, 才能真正投入临床应用. 从抗癌 的角度来说, 只要能够深入地了解某些疾病的发病机 制, 就有可能针对这一机制研发出新的治疗策略 [77-79].

\section{5 总结与展望}

大环分子具有独特的空腔结构能够有效地实现对 小分子以及离子的特异性识别与跨膜传输, 因此是构筑 新型人工跨膜离子通道的常用基元之一. 通过在大环分 子上修饰刺激响应型基团, 还可以实现对离子通道传输 性质以及开-关的智能调控, 因此大环类离子通道的研 究受到了学者们的广泛关注. 本综述详细论述了基于大 环分子衍生物的人工跨膜离子通道的构筑策略、结构调 控以及其在生物医学领域中潜在的应用价值. 尽管与天 然的通道蛋白相比, 人工离子通道具有结构简单、性能 稳定、价格低廉等一系列优点, 但是目前所报道的人工 通道也存在一些明显的问题, 例如离子的传输选择性不 高; 传输过程多为被动扩散; 人工通道还具有一定的细 胞毒性等. 因此还需要研究人员们的深入研究, 开发出 可以和天然通道相媲美的人工跨膜结构, 以期早日实现 人工离子通道在医药和化工产业中的广泛应用.

\section{作者简介}

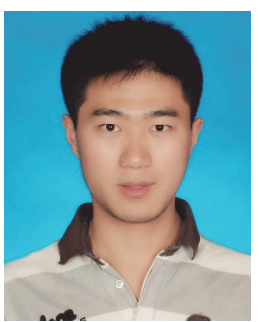

间腾飞, 博士后, 1990 年出生于河北省石家庄, 2014 年本 科毕业于廊坊师范学院化学与材料科学学院, 2019 年博士毕 业于吉林大学化学学院高分子化学与物理专业, 导师为刘俊 秋教授. 2020 年入职中南大学化学化工学院从事博士后研究 (与杭州师范大学联合培养), 导师为邹应萍教授和刘俊秋教授, 研究方向为生物超分子材料的制备与性能研究.

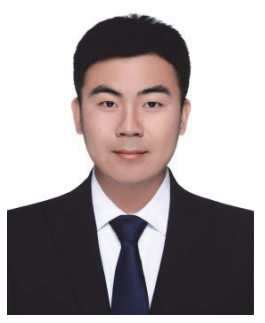

刘盛达, 博士后, 1991 年出生于吉林省吉林市, 2015 年本 科毕业于长春理工大学化学与环境工程学院, 2020 年博士毕 业于吉林大学化学学院高分子化学与物理专业, 导师为刘俊
秋教授. 2020 年入职中南大学化学化工学院从事博士后研究 (与杭州师范大学联合培养), 导师为刘又年教授和刘俊秋教授, 研究方向为仿生高分子材料.

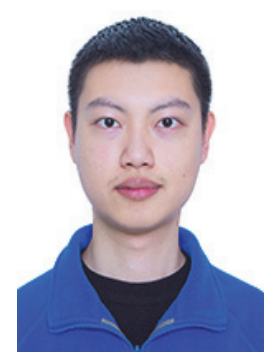

罗逸尘, 硕士, 1995 年出生于浙江省台州市, 2017 年本科 毕业于复旦大学高分子科学系, 导师为周平教授. 2020 年进入 杭戊师范大学材料与化学化工学院攻读硕士学位, 导师为刘 俊秋教授, 研究方向为新型智能离子通道的设计与开发.

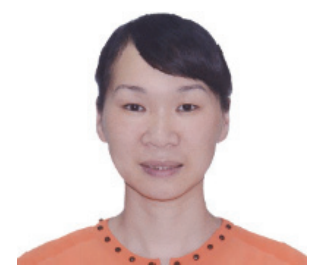

邹应萍, 中南大学化学化工学院教授, 博士生导师. 2008 年博士毕业于中国科学院化学研究所. 2008 年至今中南大学 从事教学科研工作, 2014 年 2 月破格晋升教授. 在加拿大拉瓦 尔大学(2008 2010 年)和美国斯坦福大学(2012 2014 年)从 事博士后/访问研究. 在 SCI 源刊物上发表学术论文 200 余篇, 引用 10000 余次, $\mathrm{H}$ 指数为 49 . 主要研究方向为有机高分子光 电材料与器件.

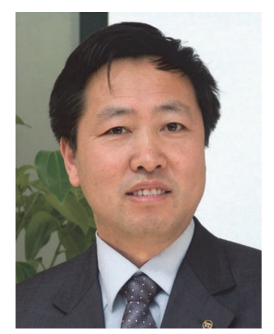

刘俊秋, 杭州师范大学材料与化学化工学院教授, 1999 年 于吉林大学化学系高分子专业获理学博士学位. 2002 2003 年获洪堡基金资助在德国从事博士后研究. 2003 年加入吉林 大学超分子结构与材料国家重点实验室，担任全职教授. 2019 年调入杭州师范大学材料与化学化工学院工作, 研究方向包 括仿生化学、蛋白质组装与功能化、生物材料等.

\section{References}

[1] Hedin, L. E.; Illergard, K.; Elofsson, A. Proteome Res. 2011, 10, 3324.

[2] Cho, W.; Stahelin, R.V. Annu. Rev. Bioph. Biom. 2005, 34, 119.

[3] Elborn, J. S. Lancet 2016, 388, 2519.

[4] Sanguinetti, M. C.; Tristani-Firouzi, M. Nature 2006, 440, 463.

[5] Sakai, N.; Sordé, N.; Matile, S. J. Am. Chem. Soc. 2003, 125, 7776.

[6] Sakai, N.; Matile, S. Langmuir 2013, 29, 9031.

[7] Guo, Y.; Zhang, Y.; Li, J.; Zhao, F.; Liu, Y.; Su, M.; Jiang, Y.; Liu, Y.; Zhang, J.; Yang, B.; Yang, R. Chin. J. Chem. 2016, 34, 425.

[8] Liu, Z.; Samanta, A.; Lei, J.; Sun, J.; Wang, Y.; Stoddart, J. F. J. Am. 
Chem. Soc. 2016, 138, 11643.

[9] Liu, Z.; Frasconi, M.; Lei, J.; Brown, Z. J.; Zhu, Z.; Cao, D.; Iehl, J.; Liu, G.; Fahrenbach, A. C.; Botros, Y. Y.; Farha, O. K.; Hupp, J. T.; Mirkin, C. A.; Fraser-Stoddart, J. Nat. Commun. 2013, 4, 1855.

[10] Tabushi, I.; Kuroda, Y.; Yokota, K. Tetrahedron Lett. 1982, 23, 4601.

[11] Anderson, M. P.; Rich, D. P.; Gregory, R. J.; Smith, A. E.; Welsh, M. J. Science 1991, 251, 679.

[12] Madhavan, N.; Robert, E. C.; Gin, M. S. Angew. Chem., Int. Ed. 2005, 44, 7584 .

[13] Mamad-Hemouch, H.; Ramoul, H.; Abou, T. M.; Bacri, L.; Huin, C.; Przybylski, C.; Oukhaled, A.; Thiébot, B.; Patriarche, G.; Jarroux, N.; Pelta, J. Nano Lett. 2015, 15, 7748.

[14] Pedersen, C. J. J. Am. Chem. Soc. 1967, 89, 7017.

[15] Tang, C. C.; Wang, L. H.; Yun, Y. B.; Zhang, C. L.; Liu, B. Q. Acta Chim. Sinica 2011, 69, 343 (in Chinese). (唐橙橙, 王丽华, 贠延滨, 张陈淋, 刘必前, 化学学报, 2011, 69, 343.)

[16] Gokel, G. W.; Leevy, W. M.; Weber, M. E. Chem. Rev. 2004, 104, 2723.

[17] Nakano, A.; Xie, Q.; Mallen, J. V.; Echegoyen, L.; Gokel, G. W. J. Am. Chem. Soc. 1990, 112, 1287.

[18] Negin, S.; Patel, M. B.; Gokel, M. R.; Meisel, J. W.; Gokel, G. W. ChemBioChem 2016, 17, 2153.

[19] Atkins, J. L.; Patel, M. B.; Cusumano, Z.; Gokel, G. W. Chem. Commun. 2010, 46, 8166.

[20] Neevel, J. G.; Nolte, R. J. M. Tetrahedron Lett. 1984, 25, 2263.

[21] Voyer, N.; Robitaille, M. J. Am. Chem. Soc. 1995, 117, 6599.

[22] Ren, C.; Shen, J.; Zeng, H. J. Am. Chem. Soc. 2017, 139, 12338.

[23] Li, N.; Chen, F.; Shen, J.; Zhang, H.; Wang, T.; Ye, R.; Li, T.; Loh, T. P.; Yang, Y. Y.; Zeng, H. J. Am. Chem. Soc. 2020, 142, 21082.

[24] Ren, C.; Zeng, F.; Shen, J.; Chen, F.; Roy, A.; Zhou, S.; Ren, H.; Zeng, H. J. Am. Chem. Soc. 2018, 140, 8817.

[25] Roy, A.; Joshi, H.; Ye, R.; Shen, J.; Chen, F.; Aksimentiev, A.; Zeng, H. Angew. Chem., Int. Ed. 2020, 59, 4806.

[26] Shen, J.; Fan, J.; Ye, R.; Li, N.; Mu, Y.; Zeng, H. Angew. Chem., Int. Ed. 2020, 59, 13328.

[27] Shen, J.; Ye, R.; Romanies, A.; Roy, A.; Chen, F.; Ren, C.; Liu, Z.; Zeng, H. J. Am. Chem. Soc. 2020, 142, 10050.

[28] Böhmer, V. Angew. Chem., Int. Ed. 1995, 34, 713.

[29] Li, C. B.; Yue, Y. L.; Liu, B. Q.; Fan, S. D. Chin. J. Org. Chem. 2011, 31, 819 (in Chinese). (李春斌, 岳玉莲, 刘宝全, 范圣第, 有 机化学, 2011, 31, 819.)

[30] Matthews, S. E.; Schmitt, P.; Felix, V.; Drew, M. G. B.; Beer, P. D. J. Am. Chem. Soc. 2002, 124, 1341.

[31] Tanaka, Y.; Kobuke, Y.; Sokabe, M. Angew. Chem., Int. Ed. 1995, 34, 693.

[32] Yoshino, N.; Satake, A.; Kobuke, Y. Angew. Chem., Int. Ed. 2001, 40, 457.

[33] Ogoshi, T.; Kanai, S.; Fujinami, S.; Yamagishi, T.-A.; Nakamoto, Y. J. Am. Chem. Soc. 2008, 130, 5022.

[34] Si, W.; Xin, P.; Li, Z.-T.; Hou, J.-L. Acc. Chem. Res. 2015, 48, 1612.

[35] Xue, M.; Yang, Y.; Chi, X.; Zhang, Z.; Huang, F. Acc. Chem. Res. 2012, 45, 1294.

[36] Si, W.; Hu, X.-B.; Liu, X.-H.; Fan, R.; Chen, Z.; Weng, L.; Hou, J.-L. Tetrahedron Lett. 2011, 52, 2484.

[37] Si, W.; Chen, L.; Hu, X.-B.; Tang, G.; Chen, Z.; Hou, J.-L.; Li, Z.-T. Angew. Chem., Int. Ed. 2011, 50, 12564.

[38] Chen, J.-Y.; Xiao, Q.; Behera, H.; Hou, J.-L. Chinese Chem. Lett. 2020, 31, 77 .

[39] Han, B.; Liu, Y. Chin. J. Org. Chem. 2003, 23, 139 (in Chinese). (韩 宝航, 刘育, 有机化学, 2003, 23, 139.)

[40] Kim, K.; Selvapalam, N.; Ko, Y. H.; Park, K. M.; Kim, D.; Kim, J. Chem. Soc. Rev. 2007, 36, 267.

[41] Lee, J. W.; Samal, S.; Selvapalam, N.; Kim, H.-J.; Kim, K. Acc. Chem. Res. 2003, 36, 621.

[42] Zhou, Y.; Morais-Cabral, J. H.; Kaufman, A.; MacKinnon, R. Nature 2001, 414, 43

[43] Jeon, Y. J.; Kim, H.; Jon, S.; Selvapalam, N.; Oh, D. H.; Seo, I.; Park, C.-S.; Jung, S. R.; Koh, D.-S.; Kim, K. J. Am. Chem. Soc. 2004, 126, 15944 .
[44] Andersen, O. S.; Koeppe, R. E.; Roux, B. IEEE T. NanoBiosci. 2005, 4, 10 .

[45] Beaven, A. H.; Maer, A. M.; Sodt, A. J.; Rui, H.; Pastor, R. W.; Andersen, O. S.; Im, W. Biophys. J. 2017, 112, 1185.

[46] Ghadiri, M. R.; Granja, J. R.; Buehler, L. K. Nature 1994, 369, 301.

[47] Montenegro, J.; Ghadiri, M. R.; Granja, J. R. Acc. Chem. Res. 2013, 46, 2955.

[48] Zhou, X.; Liu, G.; Yamato, K.; Shen, Y.; Cheng, R.; Wei, X.; Bai, W.; Gao, Y.; Li, H.; Liu, Y.; Liu, F.; Czajkowsky, D. M.; Wang, J.; Dabney, M. J.; Cai, Z.; Hu, J.; Bright, F. V.; He, L.; Zeng, X. C.; Shao, Z.; Gong, B. Nat. Commun. 2012, 3, 949.

[49] Hud, N. V.; Smith, F. W.; Anet, F. A. L.; Feigon, J. Biochemistry 1996, 35, 15383 .

[50] Kwok, C. K.; Merrick, C. J. Trends Biotechnol. 2017, 35, 997.

[51] Kaucher, M. S.; Harrell, W. A.; Davis, J. T. J. Am. Chem. Soc. 2006, $128,38$.

[52] Furukawa, H.; Kim, J.; Plass, K. E.; Yaghi, O. M. J. Am. Chem. Soc. 2006, 128, 8398 .

[53] Eisenman, G.; Horn, R. J. Membrane Biol. 1983, 76, 197.

[54] Xin, P.; Kong, H.; Sun, Y.; Zhao, L.; Fang, H.; Zhu, H.; Jiang, T.; Guo, J.; Zhang, Q.; Dong, W.; Chen, C.-P. Angew. Chem., Int. Ed. 2019, 58, 2779.

[55] Chen, J.-Y.; Hou, J.-L. Org. Chem. Front. 2018, 5, 1728.

[56] Bao, C.; Ma, M.; Meng, F.; Lin, Q.; Zhu, L. New J. Chem. 2015, 39 , 6297.

[57] Zhou, Y.; Chen, Y.; Zhu, P.-P.; Si, W.; Hou, J.-L.; Liu, Y. Chem. Commun. 2017, 53, 3681 .

[58] Abbott, G. W. Front. Physiol. 2020, 11, 583.

[59] Si, W.; Li, Z.-T.; Hou, J.-L. Angew. Chem., Int. Ed. 2014, 53, 4578.

[60] Jog, P. V.; Gin, M. S. Org. Lett. 2008, 10, 3693.

[61] Xin, P.; Tan, S.; Wang, Y.; Sun, Y.; Wang, Y.; Xu, Y.; Chen, C.-P. Chem. Commun. 2017, 53, 625.

[62] Gentzsch, M.; Mall, M. A. Chest 2018, 154, 383.

[63] Burg, S.; Attali, B. Trends Pharmacol. Sci. 2021, 42, 491.

[64] Lerche, H.; Jurkat-Rott, K.; Lehmann-Horn, F. Am. J. Med. Genet. 2001, 106, 146.

[65] Martinez, P. M.; Molenaar, P. C.; Losenn, M.; Stevens, J.; Baets, M. H. D.; Szoke, M.; Honnorat, J.; Tamouza, R.; Leboyer, M.; Os, J. V.; Rutten, B. P. F. Front. Genet. 2013, 4, 1.

[66] Muraglia, K. A.; Chorghade, R. S.; Kim, B. R.; Tang, X. X.; Shah, V. S.; Grillo, A. S.; Daniels, P. N.; Cioffi, A. G.; Karp, P. H.; Zhu, L.; Welsh, M. J.; Burke, M. D. Nature 2019, 567, 405.

[67] Béven, L.; Helluin, O.; Molle, G.; Duclohier, H.; Wróblewski, H. BBA-Biomembranes 1999, 1421, 53.

[68] Wu, C.; Li, M.; Chen, W. Diabet. Metab. Synd. Ob. 2021, 14, 285.

[69] Haoyang, W.-W.; Zhang, M.; Hou, J.-L. Chin. J. Chem. 2019, 37, 25.

[70] Leevy, W. M.; Donato, G. M.; Ferdani, R.; Goldman, W. E.; Schlesinger, P. H.; Gokel, G. W. J. Am. Chem. Soc. 2002, 124, 9022.

[71] Xin, P.; Sun, Y.; Kong, H.; Wang, Y.; Tan, S.; Guo, J.; Jiang, T.; Dong, W.; Chen, C.-P. Chem. Commun. 2017, 53, 11492.

[72] Prevarskaya, N.; Skryma, R.; Shuba, Y. Trends Mol. Med. 2010, 16, 107.

[73] Kunzelmann, K. J. Membrane Biol. 2005, 205, 159.

[74] Abdul, M.; Hoosein, N. Cancer Let. 2002, 186, 99.

[75] Britschgi, A.; Bill, A.; Brinkhaus, H.; Rothwell, C.; Clay, I.; Duss, S.; Rebhan, M.; Raman, P.; Guy, C. T.; Wetzel, K.; George, E.; Popa, M. O.; Lilley, S.; Choudhury, H.; Gosling, M.; Wang, L.; Fitzgerald, S.; Borawski, J.; Baffoe, J.; Labow, M.; Gaither, L. A.; Bentires-Alj, M. Proc. Natl. Acad. Sci. U. S. A. 2013, 110, 1026.

[76] Spugnini, E. P.; Sonveaux, P.; Stock, C.; Perez-Sayans, M.; De Milito, A.; Avnet, S.; Garcìa, A. G.; Harguindey, S.; Fais, S. BBABiomembranes 2015, 1848, 2715.

[77] Panyi, G.; Beeton, C.; Felipe, A. Philos. T. R. Soc. B 2014, 369, 20130106.

[78] Pedersen, S. F.; Stock, C. Cancer Res. 2013, 73, 1658.

[79] Haoyang, W.-W.; Xiao, Q.; Ye, Z.; Fu, Y.; Zhang, D.-W.; Li, J.; Xiao, L.; Li, Z.-T.; Hou, J.-L. Chem. Commun. 2021, 57, 1097.

(Cheng, B.) 